\section{Diseño estratégico y participativo en emprendimientos integrados. Caso fitness La Plata, Argentina ${ }^{1}$}

Strategic and participatory design in integrated ventures. Fitness case La Plata, Argentina

Cómo citar:

Del Giorgio Solfa, F. \& Alvarado Wall, T.A., 2021. Diseño estratégico y participativo en emprendimientos integrados. Caso fitness La Plata, Argentina. Designia, 9(1), 17-37.

El presente artículo responde a una etapa del Proyecto I+D Bienal 2020: “Gestión Integrada de Diseño e Innovación. Contribuciones para una revisión teórico-conceptual y metodológica". Proyecto I+D Bienal 2020. Comisión de Investigaciones Científicas y Tecnológicas. Secretaría de Ciencia y Técnica, Universidad Nacional de La Plata. Código: B374.

* Profesor en Diseño Industrial, Magister en Marketing Internacional, Profesor Titular Ordinario y Profesor Estable de Maestrías, de la Universidad Nacional de La Plata (UNLP). Especialista en Gestión Pública, Universidad Nacional de Tres de Febrero (UNTREF). Master in Diritto, Economia e Política dell'Unione Europea, Università degli Studi di Padova (UNIPD). Profesor

Titular de posgrado en la Universidad Nacional de Lomas de Zamora (UNLZ). Profesor Invitado en el Doctorado de Diseño, Universidad de Palermo (UP). Investigador Adjunto sin Director, Director de Becas Doctorales y Miembro de la Comisión Asesora Honoraria en Ingeniería, Arquitectura y Tecnología (INART), de la Comisión de Investigaciones Científicas de la Provincia de Buenos Aires (CIC-PBA). Miembro del Comité de Pares en Ciencias Aplicadas de la Comisión Nacional de Evaluación y Acreditación Universitaria (CONEAU). E-mail: delgiorgio@fba.unlp.edu.ar ORCID: https://orcid.org/0000-0002-0962-531X

\section{Palabras clave:}

Diseño estratégico, diseño participativo, emprendimientos, fitness, La Plata.

Key words:

Strategic design, participatory design, entrepreneurship, fitness, La Plata.

Recibido: 05/03/2021 
** Diseñadora Industrial, Adscripta a la cátedra de Tecnología de Diseño Industrial I-III (B) y doctoranda en Artes de la Facultad de Artes, Universidad Nacional de La Plata (UNLP). Maestranda en Marketing Internacional (EPMI) de la Facultad de Ciencias Económicas (UNLP). Becaria Doctoral con proyecto de investigación en Design Thinking, cofinanciado por la Comisión de Investigaciones Científicas de la Provincia de Buenos Aires (CIC-PBA) y la UNLP.

E-mail: twall@empleados.fba.unlp.edu.ar ORCID: https://orcid.org/0000-0002-5305-3638

\section{Resumen:}

El objetivo de este artículo es analizar las relaciones entre las teorías del diseño estratégico y el diseño participativo, en alianzas comerciales múltiples entre emprendedores locales de diversos sectores y su aplicación integrada en el contexto urbano. Diversos autores han tratado estos temas estratégicos de manera aislada y con menor frecuencia los han abordado desde la experiencia emprendedora. Una revisión de la literatura específica nos permite dar cuenta de los principales conceptos involucrados sobre este enfoque. El caso que se analiza refiere a un emprendimiento vinculado al fitness en la ciudad de La Plata, que articuló junto a otros actores locales una estrategia colaborativa que dio lugar a la aplicación de un modelo de negocios superador para su sector. Complementariamente, capitalizó su rol de influencer en la red social Instagram, como forma de potenciar la integralidad del proyecto. Los temas que se abordan sintetizan los conceptos claves que nos permiten interpretar la praxis relevada. Y a partir de ellos conformar un modelo que pueda ser aplicado en otros mercados y territorios para impulsar el desarrollo local joven. Y de esta forma distintos actores, productos y servicios complementarios sirvan para promover conjuntamente una cultura coproductiva y autogestiva. 


\begin{abstract}
:
The objective of this article is to analyze the relationships between theories of strategic design and participatory design, in multiple commercial alliances between local entrepreneurs from different sectors and their integrated application in the urban context. Various authors have dealt with these strategic issues in isolation and less frequently have addressed them from the entrepreneurial experience. A review of the specific literature allows us to account for the main concepts involved in this approach. The case being analyzed refers to a fitness-related venture in the city of La Plata, which articulated, together with other local actors, a collaborative strategy that led to the application of a superior business model for its sector. In addition, he capitalized on his role as an influencer on the social network Instagram, as a way to enhance the project's comprehensiveness. The topics that are addressed synthesize the key concepts that allow us to interpret the praxis relieved. And from them, create a model that can be applied in other markets and territories to promote young local development. And in this way, different actors, complementary products and services serve to jointly promote a co-productive and self-managed culture.
\end{abstract}

\title{
INTRODUCCIÓN
}

El presente trabajo se propone reflexionar sobre el rol estratégico y participativo del diseño en el desarrollo de las economías locales, tomando como caso de análisis un emprendimiento integrado del sector fitness en la ciudad de La Plata, Argentina. La elección de este caso se fundamenta en la relevancia creciente que está teniendo el diseño como herramienta integradora y potenciadora de diferentes acciones emprendedoras de los jóvenes en un área cohesionada del ámbito urbano (Del Giorgio Solfa, 2015).

El caso representa un modelo de alianza de diversos emprendimientos, principalmente liderados por jóvenes millennials, que son significativos en tendencia y generación de empleos como respuesta a la coyuntura socio-económica.

El desarrollo de este artículo se centrará en analizar las relaciones que existen entre las teorías del diseño estratégico y el diseño participativo, para su aplicación en las alianzas comerciales múltiples que se dan entre emprendedores locales jóvenes de diversos sectores productivos y su aplicación integrada en el contexto de la ciudad de La Plata. El objetivo es desentramar cómo el accionar estratégico del diseño puede aportar al desarrollo local, creando valor para un segmento joven del mercado que le es propio. En este abordaje, se valida la construcción de este tipo de 
relaciones productivas en red, que benefician de manera sinérgica a las actividades principal y particulares asociadas. En este tipo de trabajo en red se da un modo de accionar dinámico, que permanentemente actualiza sus respuestas acompañando el crecimiento y evolución de su mercado objetivo. El segmento del mercado al que se apunta, se caracteriza por un consumo consciente que se presta especial atención al medioambiente, la vida saludable, el origen y la forma en que se producen los insumos.

Este trabajo, pretende capitalizar este tipo de experiencia en un modelo que pueda ser fácilmente aplicable en diferentes sectores y poblaciones urbanas, como forma de desarrollo económico que apunta a un mejor balance entre empleo y calidad de vida de los jóvenes.

\section{Los elementos del diseño estratégico}

Para definir al diseño estratégico, es necesario entender el origen conceptual de la estrategia. La estrategia, que proviene del ámbito militar y político y se asume en los niveles más jerárquicos de una organización, analiza los recursos disponibles, la viabilidad de las acciones, los escenarios posibles, para fijar objetivos estratégicos que permitan alcanzar los resultados esperados (Urquilla, 2014).

En el ámbito empresarial, la estrategia que se enmarca en la misión de la empresa, tiene el propósito de alcanzar metas, creando valor a partir de la definición de cartera de negocios y productos (Levy, 2012; Mortati, Villari y Maffei, 2014; Ali y Liem, 2015). La definición de una estrategia corporativa debe dirigirse a necesidades de un segmento específico del mercado y ofrecerles un conjunto de beneficios especiales (Kotler, 2003).

Una estrategia que permanece en el tiempo genera mayores beneficios a la organización, para lo cual pueden incorporarse las $3 \mathrm{~V}$ : value target (público objetivo), value proposition (proposición de valor) y value network (red de colaboración) (Kumar, 2007).

De forma análoga el diseño estratégico, propone un enfoque sistémico y holístico del diseño, que articula soluciones para alcanzar determinados objetivos, utilizando metodologías y elementos propios de la disciplina con el fin último de impactar positivamente en la experiencia del usuario (Bayazit, 2004). 
El diseño estratégico involucra la aplicación consciente de: el enfoque metodológico, los procesos y las herramientas del diseño, para dotar a los negocios de características diferenciadoras (Sanders, 2000; Ali y Liem, 2015). Esta perspectiva, busca expandir y capitalizar la experiencia del diseño a la cultura de las organizaciones para generar innovaciones desde el punto de vista más holístico posible (Manzini, 2003).

Es así entonces, que el diseño estratégico se vale de una posición jerárquica que se ubica entre el liderazgo y la política de diseño (Gusakov, 2020). Este enfoque se basa en la selección y ajuste de las variables que debería incluir un proyecto -como pueden ser las tecnológicas, productivas, comunicacionales, de distribución y de uso- para alcanzar un determinado objetivo (Rittel y Webber, 1973).

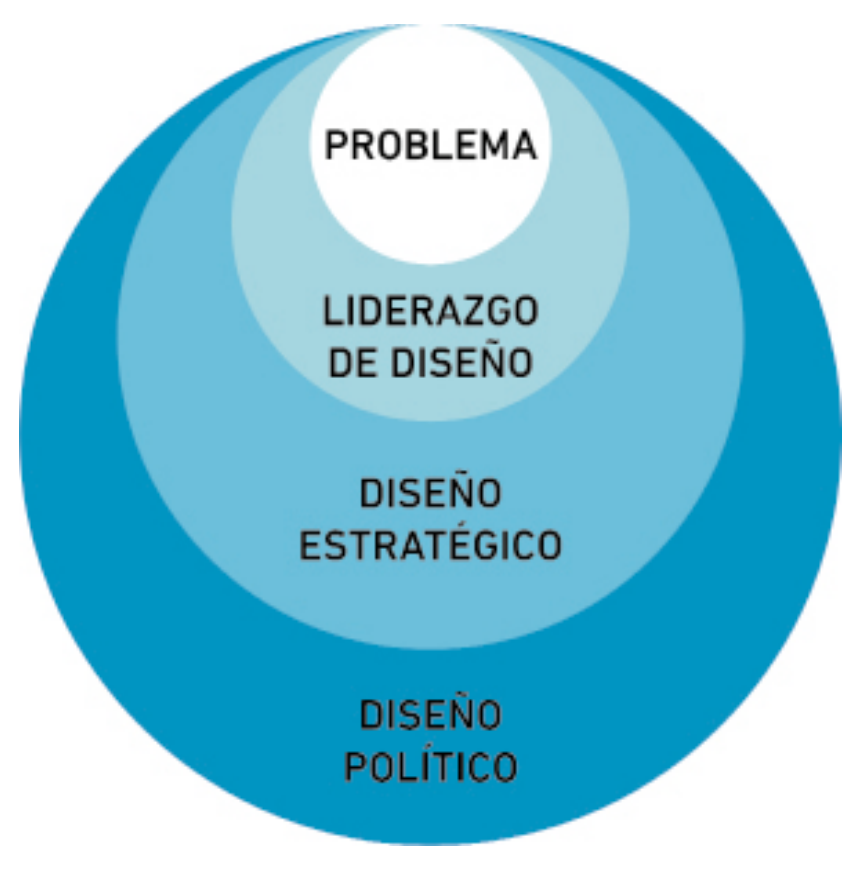

Figura 1. Jerarquía relativa del diseño estratégico. Fuente: autores. 
El análisis multidimensional que propone el diseño estratégico también es una de las claves que permite concebir integralmente todos los elementos del producto $o$ servicio en su amplio contexto para encontrar una solución acorde a la complejidad del objetivo prefijado (Arámbula Ponte y Uribe Becerra, 2016; Amendolaggine y Alvarado Wall, 2018).

El concepto de diseño estratégico nace al interno de las grandes organizaciones, como un modo de ecualizar el diseño y dar coherencia en cada uno de los elementos corporativos. Junto a la profundización de la globalización este concepto fue evolucionando y pasando de una mirada del branding corporativo a una de gestión integral de diseño (Levy, 1989; Buchanan, 1992; Kotler, 2003).

Es así que el diseño estratégico asume el liderazgo para la formulación de nuevas soluciones que consideran diferentes alternativas y convergen sinérgicamente con la filosofía empresarial (Ulloa, 2012). Este rol involucra las diferentes fases del proceso de diseño e incorpora el sistema de evaluación integral propio de la gestión estratégica (Ali y Liem, 2015; Arámbula Ponte y Uribe Becerra, 2016; Baldassarre et al., 2019). Capitalizar las metodologías del diseño en la innovación social, constituye el modo más factible para alcanzar una perspectiva sistémica y general en las acciones de la cultura emprendedora (Kvan y Candy, 2000).

En los últimos años la mirada del diseño estratégico se fue introduciendo en los pequeños emprendimientos en los cuales encontraron nuevas respuestas a necesidades puntuales en materia de diseño para potenciar su inserción en la economía local (Meroni, 2008; Macías-Loor y Macías Mendoza, 2017).

En esta perspectiva última se incorpora el concepto de diseño de servicios lo que unido a la integralidad de los elementos del diseño les da un nuevo sentido a las nuevas soluciones (Buchanan, 1992; Deserti, Meroni y Raijmakers, 2018; Del Giorgio Solfa, Amendolaggine y Alvarado Wall, 2018). 
Adiferencia del diseño estratégico, que nace de la necesidad económica de las empresas, el diseño participativo surge socialmente como un modo de accionar espontáneo que busca establecer vínculos comerciales más justos en el ámbito local (Jégou y Manzini, 2009; Liem y Sanders, 2011; Manzini, 2013).

El diseño participativo aparece como respuesta a cambios en el comportamiento de la sociedad (Cantu y Rizzo, 2012). Desde hace un tiempo el estilo de vida que propone el modelo capitalista industrializado llevó a un individualismo y consumo exacerbado poco consciente del impacto que eso genera en las sociedades y medioambiente (Vezzoli y Manzini, 2008; Jégou y Manzini, 2009).

Esta concepción de vida ha comenzado a repensarse por los individuos motivando generar una nueva cultura de consumo, más consiente, más social, más amigable con el medio ambiente. Estas nuevas formas surgen de manera vecinal pero a escala mundial y es una tendencia que se acentúa cada vez más en diversos territorios locales (Manzini, 2013).

Es por ello que el surgimiento del diseño y los sistemas participativos, han permitido el desarrollo de las capacidades humanas, motivando a los involucrados a que generen distintos aportes a la innovación, incentivados por el mismo el sistema de trabajo multidisciplinar y horizontal que se propone desde esta perspectiva de diseño (Han et al., 2012). El desarrollo de estas capacidades puede alcanzar innovación social en el sentido de mejorar la calidad de vida de las comunidades (Manzini, 2013; Del Giorgio Solfa, 2015).

En este marco, estos cambios coincidieron con la modificación del trabajo en grupo al trabajo en equipo, lo que otorgó mayor participación a los miembros y junto con el nuevo carácter transdisciplinar, permitieron una importante evolución de las soluciones arribadas en materia del diseño (Gómez Mujica y Acosta Rodríguez, 2003; Henao Villa et al., 2017).

En este orden de ideas, destacamos que la cultura participativa y coproductiva genera un sentimiento de pertenencia en las personas, que no solo los motiva e impulsa en el desarrollo personal, sino que guía y potencia los proyectos en los que cada uno se involucra (Varsavsky, 1971; Rittel y Webber, 1973; Rossi, Meroni y Selloni, 2018).

La cooperación interdisciplinaria que se da entre las partes en el diseño participativo, tiende a una mayor democratización e idea de pertenencia al sumar aceptación social, que en definitiva impactará positivamente en la viabilidad del proyecto colectivo (Llano et al., 2016; Coy y Prasch, 2019). 
Las redes sociales son clave en este tipo de cooperación para que fluya bidireccionalmente la información entre los distintos actores, reduciendo la distancia entre proyectos y destinatarios (Bocanegra Barbecho, Toscano y Delgado Anes, 2017).

Este tipo de interacción de redes, suma además espacios de creatividad que, a partir del suministro de nuevas ideas de productos y servicios, contribuyen al desarrollo de la innovación social (Cisternas, 2016). Esta última, entendida como la suma de procesos de aprendizaje y cambios en la forma en la que la sociedad diseña, produce y consume, tendiendo a modos de vida más sostenibles (Manzini, 2003; Jégou y Manzini, 2009; Rodgers, 2018).

Los modelos participativos en el espacio local, tienen como fin fomentar las relaciones de cercanía, creando y fortaleciendo vínculos entre individuos mediante organizaciones que permiten el uso compartido, el intercambio y la participación a escala vecinal (Sforzi, 2007; Sandoval Escudero, 2014).

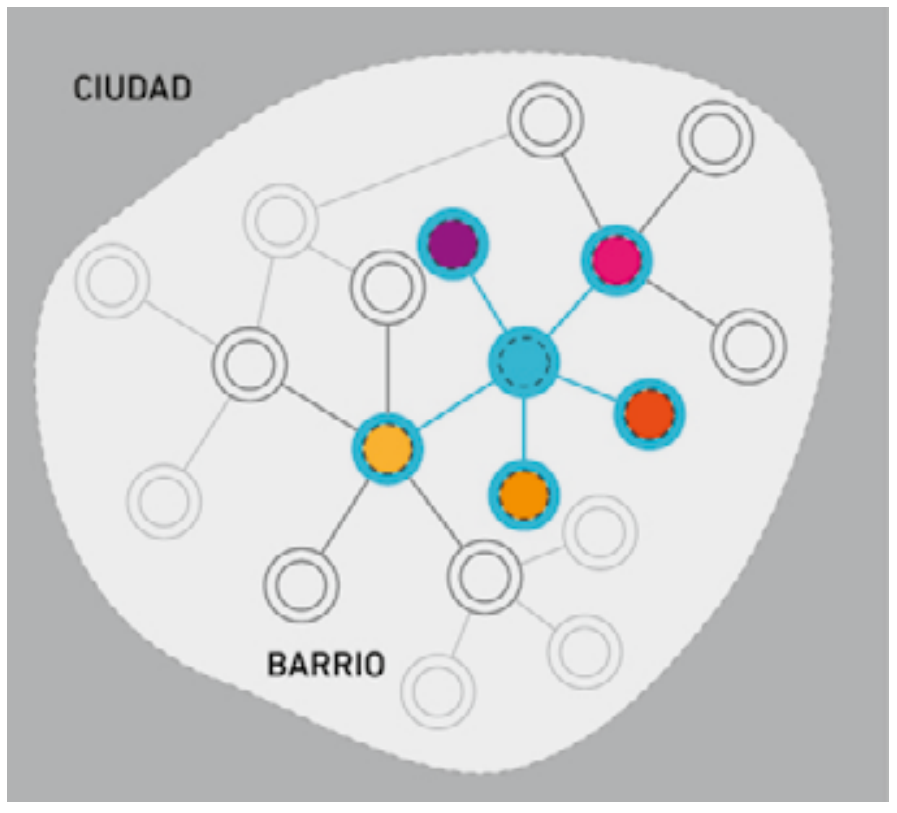

Figura 2. Red de emprendedores locales asociados. 
Para que este tipo de diseño participativo funcione, debe estar organizado y adaptado por los actores en cada contexto, apuntando a satisfacer a las demandas de un determinado grupo social (Sanders y Stappers, 2008; Manzini, 2013). Poner en funcionamiento este tipo de intervención social en materia de diseño, requiere incidir en la motivación cotidiana de las personas para que los impulse a crear espacios de interacción y competencia, que les permita entender la complejidad sistémica de los problemas locales y cuestionen el orden y las condiciones existentes (Markussen 2013; Meroni, Fassi y Simeone, 2013).

\section{El enfoque de las alianzas y emprendedores de diferentes sectores}

El diseño participativo coincide ampliamente con los intereses y posibilidades de la juventud (Molina, 2018). Etapa de la vida en la que naturalmente sus actividades adoptan un estilo de trabajo en red, encontrando soluciones a las necesidades que van surgiendo en su entorno más próximo (Smith, Bossen y Kanstrup, 2017).

También estas necesidades de distinta índole se entremezclan en la vida cotidiana de los jóvenes y tienden a encontrar respuestas integrales y periódicas, que enmarcadas en su filosofía ayudan a la organización de su rutina diaria. A su vez sus intereses y modos de consumo, tienden a buscar pequeños productores locales como contraste del consumo masivo ampliamente dominado por las multinacionales (Sepúlveda, 2002). En este razonamiento, las alianzas de emprendedores locales resultan ideales para satisfacer este tipo de demanda (Del Giorgio Solfa, 2015; Kantis, 2016).

Por otro lado, cada actividad productiva o comercial involucra en sus múltiples procesos, insumos provenientes de diferentes sectores (Verreynne, 2006). Las estrategias de logística y aprovisionamiento de la mercadería que impulsan al desarrollo local, cobran especial relevancia a la hora de la determinación de este tipo de proveedores, que en muchas ocasiones evolucionan a la categoría de alianzas (Del Giorgio Solfa y Sierra, 2016).

Al enfocarnos en los pequeños productores, se consigue adecuar metodologías y procedimientos para alcanzar un nivel de calidad superior. A su vez estos u otros emprendedores pueden suministrar otros insumos que, aunque no son parte de la actividad específica, complementan una cartera de productos y servicios que responden integralmente a un estilo de vida determinado (Jégou y Manzini, 2009). 
Estas alianzas permiten que cada emprendimiento se especialice en su área incorporando tecnologías y nuevos saberes en busca de calidad para sus productos o servicios que mejor satisfagan al público objetivo (Jevnaker y Bruce, 1998; Vargo y Akaka, 2012). Sin perder su identidad y protagonismo, los emprendedores en este accionar sinérgico del conjunto, incorporan coproducción y pequeñas innovaciones como un agregado de valor para todo el colectivo (Rossi et al., 2018; Maldonado Martínez, Estrada Gutiérrez y Sarracino Jiménez, 2020).

\section{Emprendimientos integrados en torno a un espacio multipropósito fitness}

En 2016 se inicia en La Plata (Argentina), con una sociedad joven integrada por una nutricionista y un profesor de educación física, el proyecto de Espacio de Nutrición y Actividad Física (ENAF), ante la demanda cercana del público joven que buscan mejorar su salud y calidad de vida en un mismo lugar. Al mismo tiempo que se consolidaba el ENAF, paralelamente se sumaban seguidores a la cuenta de la socia nutricionista en la red social Instagram.

En una segunda etapa, cuatro años después, el espacio fitness se desarrolla promoviendo la calidad de vida saludable, la conciencia y soberanía alimentaria, evolucionando al Centro de Entrenamiento y Nutrición Personalizado - ENAF.

Con las redes sociales incorporadas, un amplio número de seguidores y un nuevo espacio físico ampliado, se experimenta un tercer momento donde se visualizan nuevas oportunidades para integrar productos y servicios a la oferta del ENAF.

\section{El funcionamiento del espacio multipropósito}

Valiéndose del rol de influencer adquirido, la sociedad del ENAF alcanzó un nuevo estatus que le permitió adquirir en alianzas con diferentes emprendedores: la infraestructura, el diseño institucional y de interiores, el mobiliario, la vestimenta corporativa, espónsores y otros productos y servicios complementarios.

Así mismo, la comunicación del nuevo ENAF fue un factor clave para su crecimiento que, al utilizar Instagram como único canal, consiguió multiplicar la promoción y comercialización del espacio, sus productos y servicios con sus más de 60.000 jóvenes seguidores.

El ENAF como espacio multipropósito en su dimensión física y virtual, se compone de: (1) el gimnasio y actividad física, (2) el consultorio nutricional, (3) la tienda de 
alimentos saludables, (4) la indumentaria deportiva y (5) consultorio externo para profesionales de la salud. Esto se complementa con acciones en Instagram y el sitio web como medio de comercialización de los diferentes servicios que el espacio ofrece.

El diseño y la imagen corporativa ha sido una de las claves en la integración de los diferentes elementos que componen este espacio fitness. Bajo una concepción sistémica se proyectaron las identidades visuales de: ENAF, Nutrición Mili Sanz y Tienda Real Food; consideradas como las tres unidades de negocio principales.

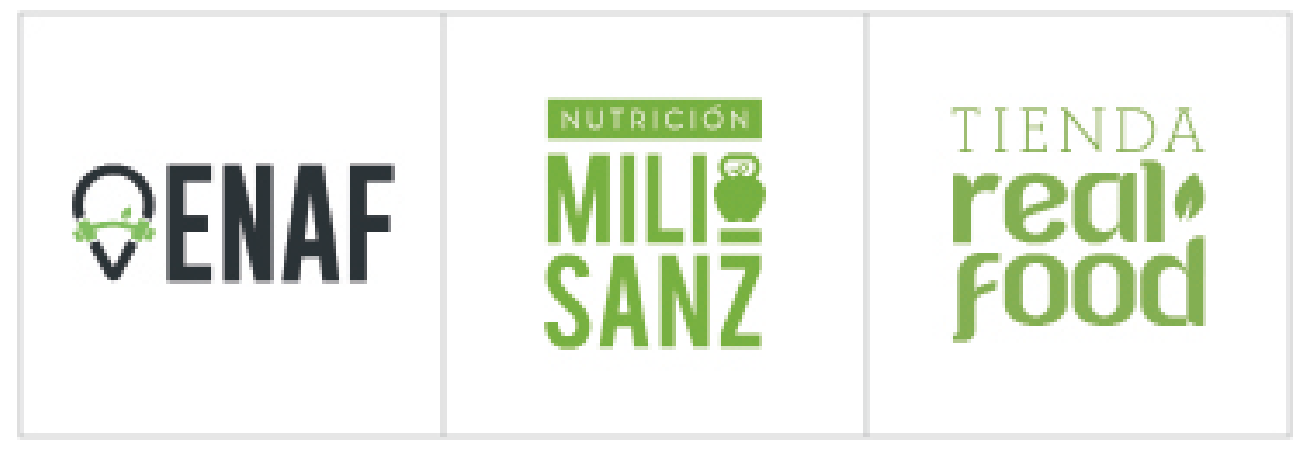

Figura 3. Sistema de identidad visual del centro ENAF.

Fuente: autores.

En cuanto al proyecto de interiorismo este alcanzó a todos los espacios físicos involucrados: recepción administrativa, consultorios, la tienda de alimentos saludables y espacio comedor, gimnasio, vestuarios y espacio social de confort. El emprendimiento dedicado a la producción de mobiliario y herrería en general, proveyó el armado de la infraestructura interna. 
Una marca de ropa deportiva local, financió la producción de murales artísticos para el interior del espacio y suministra la vestimenta corporativa de entrenadores y empleados, a su vez que ofrece a sus clientes indumentaria con la imagen del ENAF. En cuanto a los alimentos para la tienda, fueron específicamente seleccionados proveedores que sustenten la filosofía saludable. Para ello se seleccionaron productos naturales y de origen controlado, desarrollados por productores locales.

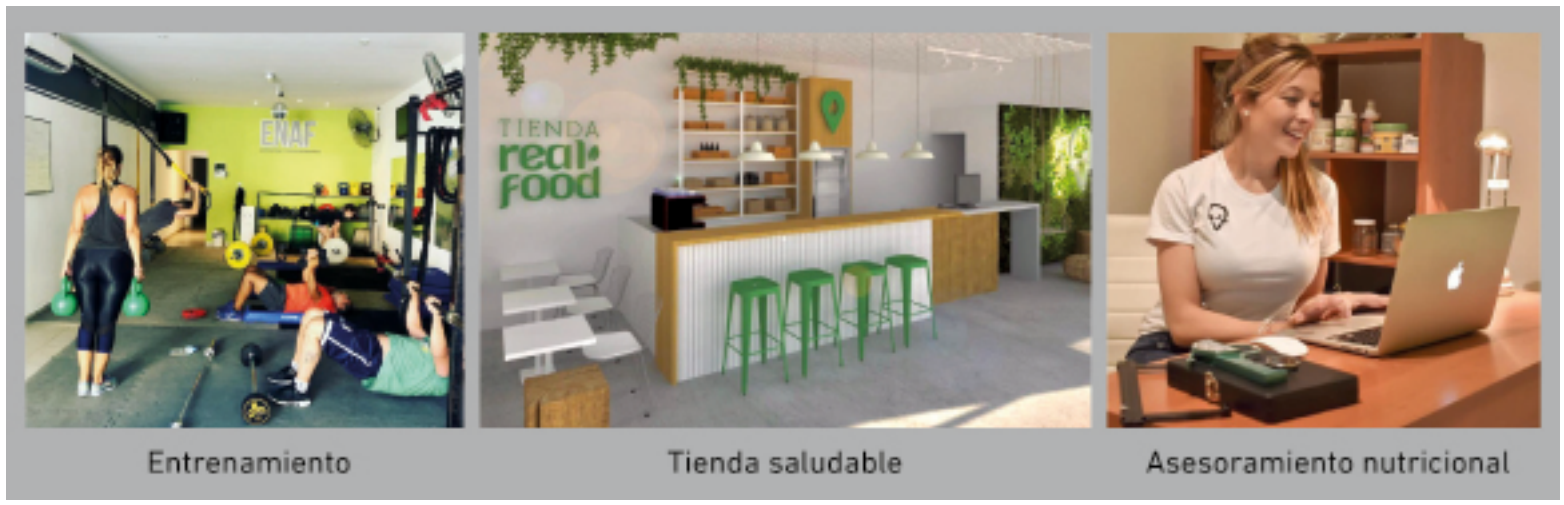

Figura 4. Imágenes del espacio integral.

Fuente: autores.

En la puesta en marcha de las unidades de negocios existió una preocupación especial en atender la demanda joven y ofrecer a los consumidores el paquete ENAF como una forma de cambio de hábitos y mejora de la calidad de vida. A los servicios de entrenamiento personalizado y consultorio nutricional ofrecidos, se incorpora la tienda de alimentos saludables completando la oferta integral. En esta tienda no solo se venden alimentos seleccionados por la nutricionista, sino que además se ofrecen distintas opciones de menú planificados bajo esta filosofía.

La novedad del modelo de negocios detectado en este caso, no solo reside en la oferta integral para los consumidores del segmento joven-fitness, sino en cómo se vinculan emprendimientos locales cercanos para el suministro de productos y servicios con diseño, coproducción y calidad controlados (Hoyer et al., 2010; Rossi, Meroni y Selloni, 2018). 
Otro punto a destacar en este análisis de caso, tiene que ver con la incorporación de las acciones estratégicas y el trabajo participativo de todos los emprendedores involucrados que potenció el impacto de sus producciones individuales llegando con un sentido alineado a una idea que el público objetivo valora (Grácio y Rijo, 2017; Menichinelli, Schmidt y Ferronato, 2019; Moras, 2019).

\section{Modelo integrador de emprendimientos para el desarrollo local joven}

Este modelo toma como base una actividad principal que permite integrar en su espacio físico y virtual a otras actividades cuyos productos y servicios lo complementan coherentemente. A su vez, estos emprendimientos secundarios y autónomos pueden integrar otras redes de trabajo, con diversa categoría y tipo de organización.

Idealmente, este modelo funciona vinculando emprendimientos de pequeña escala o que recién se inician (Verreynne, 2006). Este tipo de emprendedores se caracteriza por ser flexible y capaz de adaptarse a las necesidades dinámicas del conjunto y adicionar prácticas participativas en la determinación de su funcionamiento, sus productos y servicios (Esparragoza et al., 2015).

La implementación que inicia en torno a la actividad principal determina un radio de acción acorde a la escala urbana inmediata (barrio) (Sforzi, 2007). Dentro de esta escala en una primera instancia la actividad principal asumirá un rol de liderazgo y buscará a sus aliados estratégicos bajo el concepto integrador elegido.

Basándonos en Kotler (2003), las organizaciones que establecen estrategias diferenciadoras, se caracterizan por: (1) determinar el mercado meta y el conjunto de necesidades a satisfacer, (2) diseñar una propuesta de valor singular para el segmento y (3) valerse de un sistema de emprendedores que faciliten el desarrollo de la propuesta de valor elaborada (Mortati et al., 2014). Este sistema de emprendimientos no puede ser imitado fácilmente al menos en el mismo entorno barrial, debido a los compromisos asumidos y a las adaptaciones efectuadas para el conjunto en sus procesos y actividades (Kumar, 2007).

La puesta en funcionamiento que incluye toda su comunicación en redes sociales, favorece la incorporación espontánea de nuevos participes, que analizados estratégicamente podrían sumar valor al conjunto de la oferta (Ruiz Moreno et al., 2014). 
El éxito del modelo, consiste en definir una idea que nuclee a la actividad principal junto a otros productos y servicios que lo complementen. Haciendo foco en las necesidades particulares de los potenciales clientes, la respuesta de cartera deberá ser coherente con el estilo de vida predominante, para que éstos resulten en una satisfacción integral que simplifique la vida de los mismos.

\section{FASES DEL MODELO}

\section{IDENTIFICAR}

Analizar el conjunto de necesidades de los clientes.

2 AMPLIAR $\begin{aligned} & \text { Relevar actividades } \\ & \text { complementarias. }\end{aligned}$

3 EXPLORAR

Büscar insumos y emprendedores locales.

\section{ACORDAR}

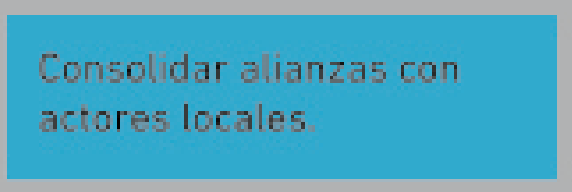

listion de sementas:

gavuest: intersol

\section{6 inNoVAR}

Aceptación de la propuesta integral en el mercado. 
Con este modelo, se busca que emprendedores puedan alcanzar una mirada general y particular sistémica, entendiendo la complejidad e interrelaciones de los diversos actores que podrían ser clave para su proyecto. También, esta capacidad podría favorecerlos en la toma de decisiones, permitiéndoles identificar y capitalizar del mejor modo los recursos disponibles a través del diseño, sin descuidar ninguna de las dimensiones esenciales del negocio que se desarrollen.

\section{Consideraciones finales}

Hasta acá hemos revisado la literatura existente y desarrollado conceptualmente los elementos de diseño estratégico, el diseño participativo y las alianzas emprendedoras entre diversos sectores.

Con un marco conceptual establecido, analizamos un caso de emprendimiento del área fitness, que involucra alianzas de diversa índole. Esto nos permitió comprender desde un nivel micro, su nacimiento, evolución y actual funcionamiento que, valiéndose de una red de actores determinada y con la incorporación de los elementos del diseño estratégico, da respuestas integrales agregando valor al mercado objetivo.

Este caso pone al descubierto una manera efectiva de como el trabajo participativo con una mirada estratégica puede potenciar el desarrollo de distintos emprendedores locales, englobados en lo que se conoce como "factores de frontera" (Leiro, 2006). También esta perspectiva responde al design thinking, logrando optimizar un pensamiento complejo en donde todo está relacionado e interconectado y cualquier actor involucrado puede ayudar a la resolución de un problema (Arámbula Ponte y Uribe Becerra, 2016; Del Giorgio Solfa et al., 2018). Así mismo se destaca el enfoque antropocéntrico que tomaron los emprendedores, detectando necesidades insatisfechas en la comunidad más próxima y madurando una solución integral, en base a la escucha e interacción permanente que les permitió decodificar aquello que no está explícito (Liem y Sanders, 2013).

Reconociendo que los emprendedores por lo general no asumen una perspectiva estratégica acorde al negocio iniciado, pero frecuentemente poseen una actitud abierta y permeable a nuevos conocimientos, asiste a la posibilidad de complementar sus saberes con nuevos conceptos que incorporan al diseño estratégico en el conjunto de herramientas para la innovación, en un mercado y región determinados. 
Para ello, se elabora y propone un modelo de fácil aplicación proyectado en seis fases, que incorpora conscientemente al diseño estratégico y responde a las necesidades de la gestión participativa para diversos actores asociados de la comunidad joven, que asumen desafíos emprendedores para el desarrollo local (Alvarado Wall, 2019).

El modelo propuesto, apunta a promover el armado de redes de emprendedores y tiene como fin consolidar una cultura coproductiva y autogestiva a escala barrial (Rossi et al., 2018). Con una cultura arraigada, las estrategias del conjunto generarán efectos positivos perfeccionando la estrategia individual de cada actor en lo que refiere a identidad, comunicación y calidad de los productos y servicios ofrecidos.

El diseño como actividad integradora será un actor clave y junto a la actividad central asumirán roles estratégicos para que en el accionar participativo, se produzcan innovaciones que impactarán integralmente a cada emprendedor del grupo asociado.

Esta nueva mirada socioeconómica intenta poner a la luz el funcionamiento de un modelo que según se observa en el caso analizado, tiene diversas ventajas para relacionar emprendedores y generar empleo autogestionado por jóvenes en el tejido productivo local.

Se espera que este artículo y el modelo propuesto ayuden a comprender integralmente las dimensiones del diseño estratégico, para que cada emprendedor pueda aplicarlas a su medida. 


\section{REFERENCIAS BIBLIOGRÁFICAS}

Ali, A. y Liem, A. (2015). The use and value of different co-creation and tools in the design process. In DS 80-3 Proceedings of the 20th International Conference on Engineering Design (ICED 15) Vol 3: Organisation and Management, Milan, Italy, 27-30.07. 15 (pp. 279-288).

Alvarado Wall, T. A. (2019). Jóvenes y diseño industrial: los nuevos contextos de inserción laboral y su relación con los diseñadores industriales jóvenes. In IX Jornadas de Investigación en Disciplinas Artísticas y Proyectuales (JIDAP) (La Plata, 22 y 23 de agosto de 2019).

Amendolaggine, G. y Alvarado Wall, T. (2018). Nuevos modelos de diseño participativo. Resolviendo problemáticas complejas. Tableros, (9), 13-23. Recuperado a partir de http://papelcosido.fba.unlp. edu.ar/ojs/index.php/tableros/article/view/755

Arámbula Ponte, P. y Uribe Becerra, M. (2016). Entendiendo el proceso de diseño desde la complejidad. Kepes, 13(13), 171-195.

Baldassarre, B. R., Calabretta, G., Bocken, N. M. P., Diehl, J. C. y Duygu, K. (2019). The evolution of the Strategic role of Designers for Sustainable Development. In Conference Proceedings of the Academy for Design Innovation Management (Vol. 2, No. 1, pp. 806-820).

Bayazit, N. (2004). Investigating design: A review of forty years of design research. Design Issues, 20(1), 16-29.

Bocanegra Barbecho, L., Toscano, M. y Delgado Anes, L. (2017). Co-creación, participación y redes sociales para hacer historia. Ciencia con y para la sociedad. Hist Comun Soc, 22(2), 325-346.

Buchanan, R. (1992). Wicked problems in design thinking. Design Issues, 8(2), 5-21.

Cantu, D. y Rizzo, F. (2012). Managing innovation through participatory processes. In E. Bohemia, J. Liedtka, \& A. Rieple (Eds.), Leading Innovation through Design: Proceedings of the DMI 2012 International Research Conference (pp. 585-593). Boston: Design Management Institute. ISBN: 978-0-615-66453-8.

Cisternas, S. F. D. (2016). El Diseño Industrial; las fronteras confusas de la creatividad. Ciencia, docencia y tecnología, 27(53), 242-361. 
Coy, E. J. y Prasch, J. E. (2019). Enhancing Collaboration. In Conference Proceedings of the Academy for Design Innovation Management, 2(1), 569-580. https://doi.org/10.33114/adim.2019.04.215

Del Giorgio Solfa, F. (2015). Juventud, cohesión social e innovación para el desarrollo local argentino: un análisis oportuno de la experiencia italiana en la Región Emilia-Romagna. Revista de Estudios Políticos y Estratégicos, 3(2), 36-49.

Del Giorgio Solfa, F. y Sierra, M. S. (2016). Contributions to Inclusive Economic Growth in Argentina: Integrating Design, Marketing and Entrepreneurship for Local Development in Buenos Aires Province. In R. Sengupta (Ed.), Pursuing Competition and Regulatory Reforms for Achieving Sustainable Development Goals (pp. 122-144). Jaipur: CUTS Internacional. ISBN13: 978-81-8257-244-7. DOI: 10.13140/RG.2.1.2102.6808.

Del Giorgio Solfa, F., Amendolaggine, G. y Alvarado Wall, T. A. (2018). Nuevos paradigmas para el diseño de productos. Design Thinking, Service Design y experiencia de usuario. Arte e Investigación, (14), e012. https://doi.org/10.24215/24691488e012

Deserti, A., Meroni, A. y Raijmakers, B. (2018). Learning and practicing in service design. In ServDes. 2018 Proof of Concept (pp. 1-12). Linköping University Electronic Press.

Esparragoza, I. E., Farak, S. L., Ocampo, J. R., Segovia, J. N., Vigano, R., Duque Rivera, J. y Rodriguez, C. A. (2015). Assessment of students' interactions in multinational collaborative design projects. International Journal of Engineering Education, 31(5), 1-15.

Gómez Mujica, A. y Acosta Rodríguez, H. (2003). Acerca del trabajo en grupos o equipos. Acimed, 11(6).

Grácio, H. L. y Rijo, C. (2017). Design thinking in the scope of strategic and collaborative design. Strategic Design Research Journal, 10(1), 30-35.

Gusakov, A. (2020). The Role of Design Thinking for Achieving Leadership in the Digital Transformation of Business. In 6th International Conference on Social, economic, and academic leadership (ICSEAL-6-2019) (pp. 356-362). Atlantis Press.

Han, K., Oh, W., Im, K. S., Chang, R. M., Oh, H. y Pinsonneault, A. (2012). Value cocreation and wealth spillover in open innovation alliances. MIS Quarterly, 291-315.

Henao Villa, C. F., Arango, D. A. G., Mesa, E. D. A., González García, A., Bracho Aconcha, R., Solorzano Movilla, J. G. y Lopez, A. P. A. (2017).

Multidisciplinariedad, interdisciplinariedad y transdisciplinariedad en la formación para la investigación en ingeniería. Revista Lasallista de investigación, 14(1), 179-197. 
Hoyer, W. D., Chandy, R., Dorotic, M., Krafft, M. y Singh, S. S. (2010). Consumer cocreation in new product development. Journal of service research, 13(3), 283-296.

Jégou, F. y Manzini, E. (2009). Servicios participativos. Experimenta: Diseño, arquitectura, comunicación, (63), 45-76.

Jevnaker, B. H. y Bruce, M. (1998). Design alliances: The hidden assets in management of strategic innovation. The Design Journal, 1(1), 24-40.

Kantis, H. (2016). La promoción del emprendimiento juvenil: su importancia para América Latina. Pensamiento Iberoamericano, 120-130.

Kotler, P. (2003). Marketing insights from A to Z: 80 concepts every manager needs to know. John Wiley \& Sons.

Kumar, N. (2007). From the $4 \mathrm{P}^{\prime}$ 's to the 3V's. The Marketer, 33, 6-9.

Kvan, T. y Candy, L. (2000). Designing collaborative environments for strategic knowledge in design. Knowledge-Based Systems, 13(6), 429-438.

Leiro, R. J. (2006). Diseño: estrategia y gestión. Buenos Aires: Infinito.

Levy, A. R. (1989). El cómo y el por qué: un camino hacia el desarrollo empresario. Buenos Aires: Tesis.

Levy, A. R. (2012). Penta innovación. Buenos Aires: Consejo Profesional de Ciencias Económicas de la Ciudad Autónoma de Buenos Aires.

Liem, A. y Sanders, E. B. N. (2013). Human-centred design workshops in collaborative strategic design projects: An educational and professional comparison. Design and Technology Education: an International Journal, 18(1), 72-89.

Liem, A. y Sanders, E. B. N. (2011, July). The impact of human-centred design workshops in strategic design projects. In International Conference on Human Centered Design (pp. 110-119). Springer, Berlin, Heidelberg.

Llano Arana, L., Gutiérrez Escobar, M., Stable Rodríguez, A., Núñez Martínez, M., Masó Rivero, R. y Rojas Rivero, B. (2016). La interdisciplinariedad: una necesidad contemporánea para favorecer el proceso de enseñanza aprendizaje. MediSur, 14(3), 320-327.

Macías Loor, A. J. y Macías Mendoza, A. J. (2017). Innovación, esquema y sostenibilidad general: nuevas tendencias para el impulso local en la modernidad. Polo del Conocimiento, 2(2), 107-127. 
Maldonado Martínez, M. M., Estrada Gutiérrez, I. y Sarracino Jiménez, K. N. (2020). Retención y atracción de clientes en empresas de servicios profesionales a través del diseño del Customer Journey Map como herramienta de Design Thinking en la mejora de la experiencia de consumo. Revista de Desarrollo Sustentable, Negocios, Emprendimiento y Educación, (mayo).

Manzini, E. (2003). Strategic Design for Sustainability: instruments for radically oriented innovation. In: Jégou, F. y Manzini, E. Sustainably everyday: scenarios of urban life. Milano: Edizioni Ambiente.

Manzini, E. (2013). Resilient systems and cosmopolitan localism - The emerging scenario of the small, local, open and connected space. Economy of Sufficiency, 70.

Markussen, T. (2013). The disruptive aesthetics of design activism: enacting design between art and politics. Design Issues, 29(1), 38-50.

Menichinelli, M., Schmidt, A. G. S. y Ferronato, P. (2019, November). Mapping strategies for distributed, social and collaborative design systems of makers, designers and social entrepreneurs. In Conference Proceedings of the Academy for Design Innovation Management (Vol. 2, No. 1, pp. 410-424).

Meroni, A. (2008). Strategic design: where are we now? Reflection around the foundations of a recent discipline. Strategic Design Research Journal, 1(1), 31-38.

Meroni, A., Fassi, D. y Simeone, G. (2013). Design for Social Innovation as a form of Design Activism: An action format. In Social Frontiers: The next edge of social innovation research conference proceedings (pp. 14-15).

Molina, D. H. (2018). Diseño participativo como instrumento para fomentar la enseñanza experimental de estudiantes de arquitectura. AREA-Agenda de Reflexión en Arquitectura, Diseño y Urbanismo, 327-345.

Moras, J. M. (2019). Experiencias pedagógicas en el ámbito del diseño para la transición. Estudio de un caso: El proyecto de comunicación del Espai Quiró. Kepes, 16(19), 217-251.

Mortati, M., Villari, B. y Maffei, S. (2014). Design Capability for value creation. In 9th DMI: Academic Design Management Conference (pp. 2490-2512).

Rittel, H. W. y Webber, M. M. (1973). Dilemmas in a general theory of planning. Policy sciences, 4(2), 155-169.

Rodgers, P. A. (2018). Design Research for Change: A UK Perspective. Diseña, (13), 110-139.

Rossi, M., Meroni, A. y Selloni, D. (2018). Massive Codesign: A Proposal for a Collaborative Design Framework. Milán: Franco Angeli. 
Ruiz Moreno, A., Ortega Egea, T., Haro Domínguez, C. y Roldán Bravo, M. (2014). El proceso de co-creación de valor y su impacto en la estrategia de innovación en empresas de servicios. Intangible Capital, 10(2), 266-293.

Sanders, E. B. N. (2000). Generative Tools for Co-designing. In: Scrivener S.A.R., Ball L.J., Woodcock A. (eds) Collaborative Design, (pp. 3-12). Springer, London. https://doi.org/10.1007/978-1-4471-0779-8 1

Sanders, E. B. N. y Stappers, P. J. (2008). Co-creation and the new landscapes of design. Co-design, 4(1), 5-18.

Sandoval Escudero, C. (2014). Métodos y aplicaciones de la planificación regional y local en América Latina. Serie Desarrollo Territorial de la CEPAL, 17, 1-85.

Sepúlveda, S. (2002). Desarrollo sostenible microregional: métodos para la planificación local. San José (Costa Rica): AgroAmérica.

Sforzi, F. (2007). Del distrito industrial al desarrollo local. En: Rosales, R. (coordinador). Desarrollo local: teoría y prácticas socioterritoriales. (pp. 27-50). México: UAM - Iztapalapa y Miguel Ángel Porrúa.

Smith, R. C., Bossen, C. y Kanstrup, A. M. (2017). Participatory design in an era of participation. CoDesign, 13(2), 65-69, http://dx.doi.org/10.1080/15710882.2017.1310466

Ulloa, I. J. F. (2012). El rol del liderazgo estratégico en las organizaciones. Saber, Ciencia y Libertad, 7(1), 119-123.

Urquilla, A. (2014). Diseño Estratégico: Motor de innovación y transformación organizacional. Anuario de investigación edición especial, 7, 9-31.

Vargo, S. L. y Akaka, M. A. (2012). Value cocreation and service systems (re) formation: A service ecosystems view. Service Science, 4(3), 207-217.

Varsavsky, O. (1971). Proyectos nacionales: Planteo y estudios de viabilidad. Buenos Aires: Ediciones Periferia.

Verreynne, M. L. (2006). Strategy-making process and firm performance in small firms. Journal of Management E Organization, 12(3), 209-222.

Vezzoli, C., y Manzini, E. (2008). Evolution of Sustainability in Design Research and Practice. Design for Environmental Sustainability, 253-262. 